\title{
An Expedited Phenotypic Approach Towards Organic Reaction Generality
}

Cesar N. Prieto Kullmer, ${ }^{1 *}$ Jacob A. Kautzky, ${ }^{1 *}$ Shane W. Krska, ${ }^{2}$ Timothy Nowak, ${ }^{3}$ Spencer D. Dreher, ${ }^{2 \dagger}$

David W. C. MacMillan ${ }^{1 \dagger}$

${ }^{1}$ Merck Center for Catalysis at Princeton University, Princeton, New Jersey 08544, USA.

${ }^{2}$ Department of Process and Analytical Chemistry, Merck Research Laboratories, Merck and Co. Inc., Rahway, NJ 07065, USA.

${ }^{3}$ Department of Discovery Chemistry, Merck Research Laboratories, Merck and Co. Inc., Kenilworth, NJ 07033, USA.

*these authors contributed equally: C.N.P.K., J.A.K.

${ }^{\dagger}$ corresponding authors: D.W.C.M (dmacmill@princeton.edu), S.D.D. (spencer_dreher@merck.com)

\section{Abstract:}

Reaction generality is crucial in determining the overall impact and usefulness of organic synthetic methods. In contrast, contemporary generalization processes seem unable to meet the growing demand for robust methodology. We sought to develop an accelerated approach towards achieving generality, inspired by phenotypic screening, that rapidly expands the scope and utility of synthetic methods. This approach was validated by example of the metallaphotoredox decarboxylative arylation, resulting in the discovery of a novel additive that overcomes many lingering limitations of this method and has significant mechanistic implications for nickel-catalyzed cross couplings in general.

Main Text:

Over the last century, advancements in organic chemistry have enabled the discovery of countless synthetic methods that have streamlined access to high-value molecular motifs. Despite the rapidly growing body of known organic transformations, few of them possess the requisite robustness and generality to impact organic synthesis and adjacent fields at large (Fig 1A). More importantly, achievement of reaction generality typically requires years to decades of specialized and costly research (1-6). This stands in stark contrast to the ever-growing need of practicing synthetic chemists to expediently amplify their repertoire of robust synthetic methods. It becomes apparent that new approaches towards reaction generalization are needed. Herein, we report the successful development of an alternative, expedited approach inspired by explorative methods employed within biology and drug discovery, specifically phenotypic screening (7-11).

Unlike target-based drug design where candidate selection is predicated on an underlying understanding of the disease in question, phenotypic screening obviates complete mechanistic understanding by applying libraries of chemically diverse compounds to entire biological systems of interest while looking for desirable changes at the phenotypic level. The advantage of phenotypic screening lies in its potential to uncover and modulate hitherto 
unrecognized biomolecular interactions, which has proven invaluable in developing powerful therapeutic approaches that leverage novel mechanisms of action and improve indications that otherwise have proven recalcitrant to traditional drug discovery techniques (12). While widely embraced in drug discovery and biology, phenotypic screening remains underutilized and unapplied within organic chemistry to date (13-15).

We realized that we could draw parallels from drug design to the problem of reaction generality: traditional approaches first seek to elucidate the mechanism of a reaction and then

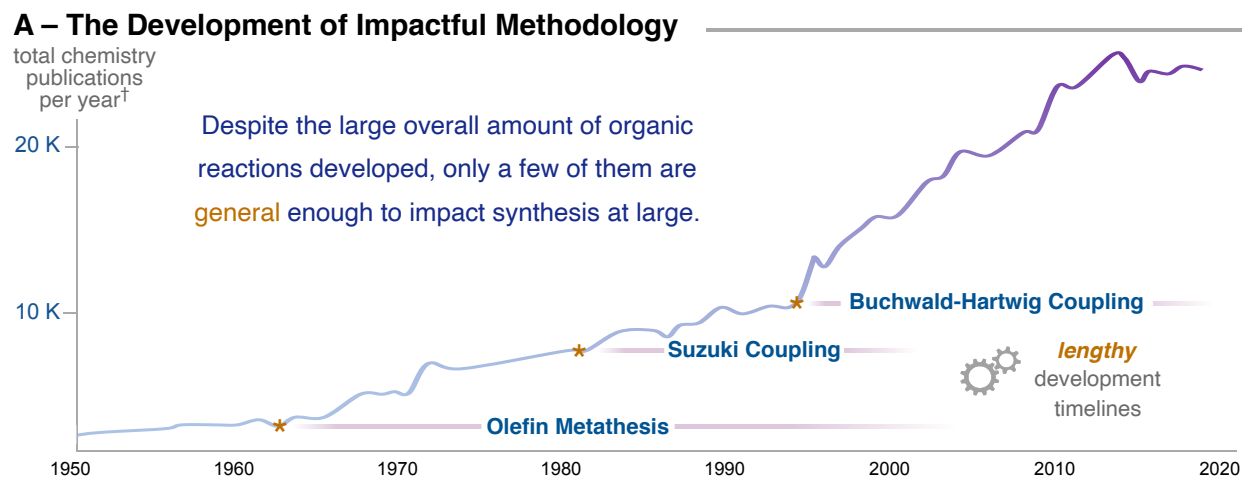

B - Reaction Generalization And This Work

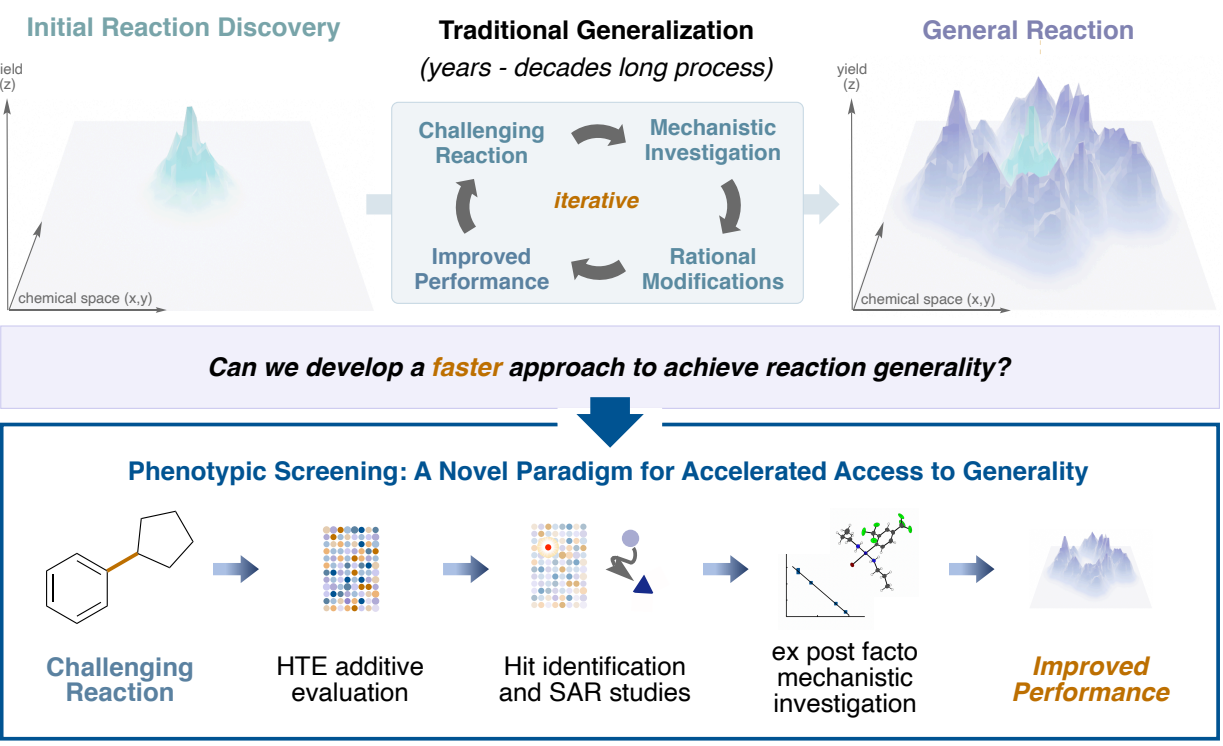

Figure 1 | Novel High-throughput Method for Improving Reaction Generality. (A) Despite considerable method development, few are general enough. (B) Traditional methods for attaining reaction generality vs. phenotypic screening.

to rationally modify parameters to achieve improvements, which mirrors target-based drug discovery (Figure 1, middle). While this process has yielded stunning successes, perhaps most notably the Buchwald-Hartwig reaction $(16,17)$, many reactions have been unable to attain generality through this process as either their mechanism is too opaque to study, mechanistic insights failed to yield any leads that could result in increased robustness, or the process proved too time intensive and costly to complete.

Phenotypic screening could prove a powerful complementary approach that complements the traditional paradigm of reaction generalization (Figure 1, bottom). In particular, this approach first undertakes a systematic unbiased modification of reaction parameters while looking for desirable changes in reaction yield for challenging substrates (the "phenotype"). Identified hits can then be further evaluated via structure-activity relationship (SAR) and mechanistic studies to identify ideal reaction conditions and understand broader chemical impacts. We envisaged that such an approach could generate unexpected and orthogonal insights that could be applied to organic methodology at large.

We turned our attention to the evaluation of additives, a generally underexplored reaction parameter. Unexpected improvements of organic reactions upon addition of unconventional additives are a well-documented 
phenomenon within organic chemistry. Examples include the lithium chloride effect in Stille couplings (18) and, more recently, work conducted by the Watson (19), Dong (20), and our own groups (21). Treating a challenging reaction with a range of chemically diverse additives could accordingly entail unforeseen mechanistic implications and lead to improved reaction performance.

For validation, the phenotypic approach was applied to the development of a synthetic method that accomplishes the long sought-after "escape from flatland" within contemporary drug discovery (22). While the likelihood of clinical success is highly correlated to the degree of saturation in a molecule, the average percentage A - Selected Challenging Reaction

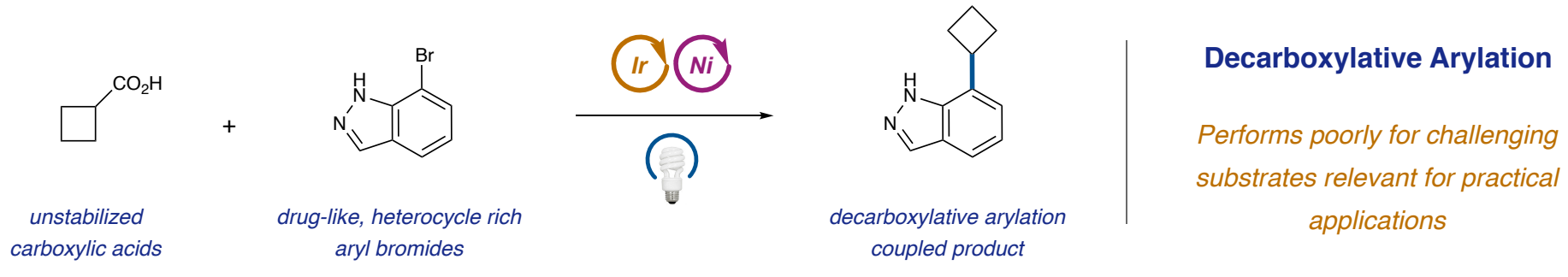

\section{B - Rapid Reaction Generality via Phenotypic Screening}

2<smiles>O=C(O)C1CCCCC1</smiles>

carboxylic acid

(+ 1 other)

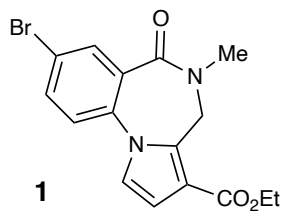

aryl halide

(+ 3 others)

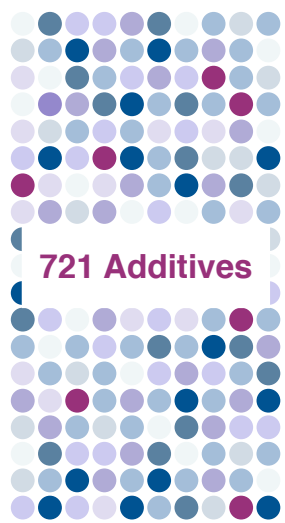

HTE Screen of

Additives

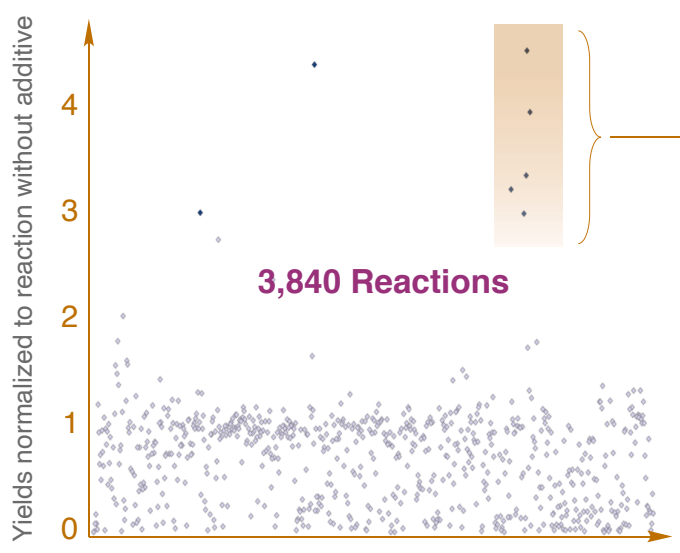

Reaction Assessment \& Analysis for Phenotypic Results (Yield)

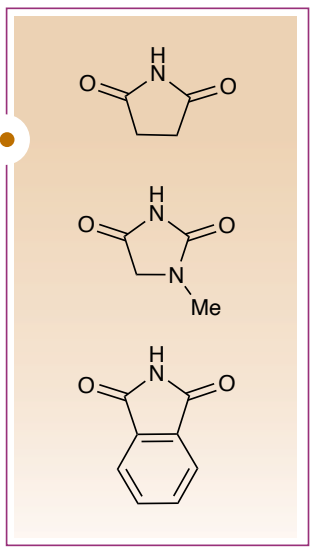

5-membered imides improved yields

\section{C - Identification of Phthalimide Additive}

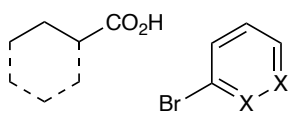

48 combinations

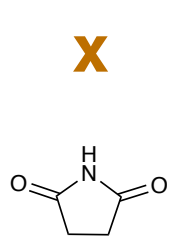

64 imide additives

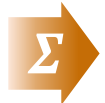

tolerated nitrogen functionalization deleterious

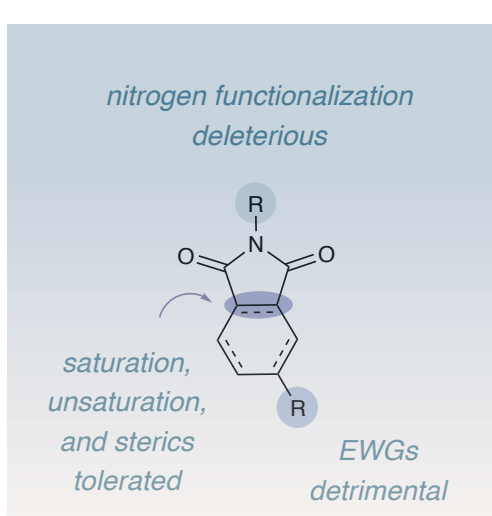

ran

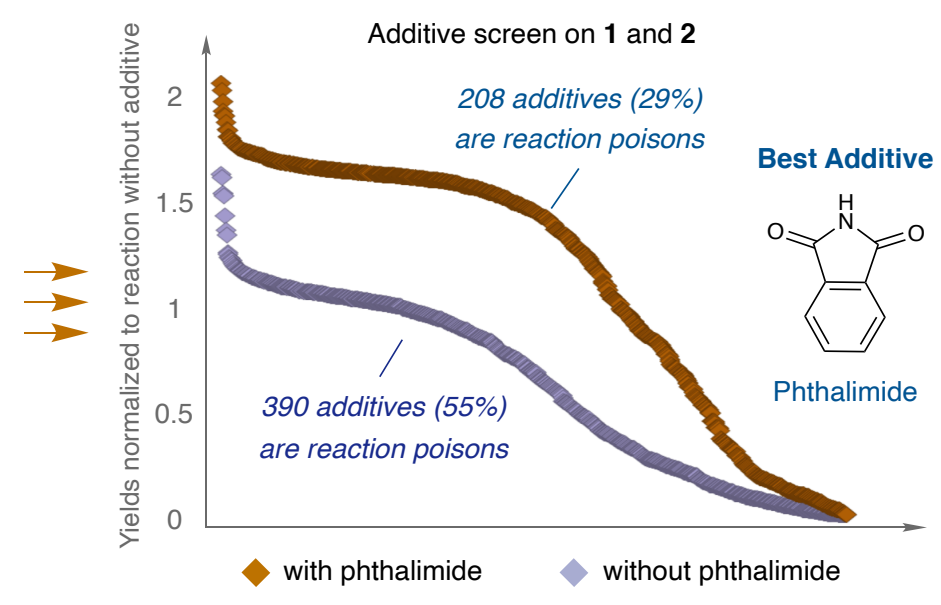

Figure 2 | Phenotypic screening applied to the decarboxylative reaction. (A) Metallaphotoredox decarboxylative arylation (B) Highthroughput additive screening on challenging reactions (C) SAR studies to identify the ideal 5-membered imide. 
of saturated carbons in approved drug molecules has fallen from 39\% in 1959-1963 to 35\% in 2005-2009 (23), which can be rationalized by the current lack of robust methodology for facile alkyl incorporation. One attractive approach is via the formation of $\mathrm{Csp}^{2}-\mathrm{Csp}^{3}$ bonds for which several elegant methods have been developed by the Buchwald, Molander, and Fu groups among others (24-29). While highly desirable, the generality of this chemistry pales in comparison to that of Nobel Prize winning $\mathrm{Csp}^{2}-\mathrm{Csp}^{2}$ cross-couplings published by Suzuki, Negishi, and Heck $(30,31)$. Few robust and operationally simple approaches towards $\mathrm{Csp}^{2}-\mathrm{Csp}^{3}$ bond formation exist today.

We envisioned that the generalization of the metallaphotoredox decarboxylative arylation initially published by the Doyle and MacMillan groups could fill this gap (30). This reaction is as a prototypical example of a green, potentially empowering transformation that hasn't been fully adopted in drug discovery despite its potential for enabling disconnections that could streamline synthesis and the broad commercial availability of both alkyl carboxylic acids and aryl halides (Fig. 2A). This can be attributed to shortcomings in reaction generality, in particular towards coordinating substrates, aryl bromides prone to protodehalogenation or with challenging oxidative additions, and non-activated carboxylic acids (acids that result in unstabilized radicals upon oxidative decarboxylation) (33). Traditional optimization of standard reaction parameters (i.e., ligand design, bases, etc.) by our group has failed to significantly expand the scope of this reaction (34). As such, phenotypic screening seemed particularly equipped to address this challenge.

To begin, we curated a library of 721 diverse organic additives with a broad coverage of chemical space (Figure 2B, also see SI). We selected several challenging coupling partners including substrates with coordinating basic nitrogens, non-activated carboxylic acids, and aryl halides that lead to sizeable quantities of Minisci and protodehalogenation side-products. These reactions were carried out in a nanoscale photoredox setup, whereby their performance, as determined by reaction yield, was evaluated in the presence of each additive. Unsurprisingly, the majority of additives and, in particular, additives containing coordinating groups such as heterocycles, anilines, and phenols significantly retarded the efficacy of the reaction (Figure 2B). Excitingly however, we discovered that various 5-membered cyclic imides and hydantoins gave a strong boost in overall yield, sometimes up to five-fold, as well as a sharp decrease in protodehalogenation. This result is highly unusual given that coordinating functionality is traditionally a powerful reaction poison due to catalyst chelation, yet here it seemed to provide beneficial effects.

Further evaluation of these unexpected hits was done via SAR studies. A total of 48 substrate combinations (3 acids against 16 aryl bromides) were selected and evaluated on a nanoscale against 64 commercially available imide and hydantoin additives (Figure 2C, left, also see Figure S10 and S11). Substrate-dependent improvements were seen for a range of additives, yet phthalimide proved to be the most broadly applicable and generally gave the largest improvement. Notably, functionalization on the nitrogen, installation of electron deficient substituents on the aromatic ring or alteration of the 5-membered ring size impaired or completely ablated the observed 
improvement. The steric effects of the imide additive though proved unimportant with even tetramethylsuccinimide performing well.

In order to gauge improvements in the functional group tolerance, compound X2 and cyclohexanoic acid were resubjected to the earlier additive screen in the presence of phthalimide, and overall reaction performance was significantly better (Figure 2C, right). A range of compounds that previously served as reaction poisons (1,3dicarbonyls, benzoic acids, etc.) were now well tolerated. Furthermore, examining the data in aggregate showed the overall average yield nearly doubled in the presence of phthalimide and the overall number of reaction poisons (defined as decreasing the yield by more than 33\%) fell from 390 to 208.

With these exciting results in hand, we sought to benchmark the reaction improvement in a pharmaceutically relevant context against the Aryl Halide Informer Library (35). This library is prototypical of the type of complex drug like-compounds seen in medicinal chemistry and contains a range of (hetero)aryl halides deemed to be inherently challenging for metal-catalyzed cross couplings. Gratifyingly, when evaluating the library against a non-activated acid we found that phthalimide had an outstanding impact. A sizeable 11 of the 18 aryl halides show noticeable performance improvements and a tripling of overall average yield was observed, from $7.7 \%$ to 29.4\% (Figure 3A, also see Figure S12). It is noteworthy that failures include compounds with free carboxylic acids (X7, X9) and aryl chlorides (X16 to 18), substrates that lie outside of the scope of this transformation. The results position the decarboxylative arylation among the most successful couplings evaluated against the library.

Encouraged by the informer results, we then set our sights on evaluating the new scope of the reaction. A highly diverse array of 384 small, medicinally relevant aryl bromides was evaluated against a complex, nonactivated isonipecotic acid derivative on a nanoscale (see SI for details). In order to gauge the synthetic utility of the phthalimide additive, we employed Corona ${ }^{\mathrm{TM}}$ charged aerosol detection (CAD) as a proxy for reaction yield (36). A 10\% reaction yield was selected as threshold for the potential isolability of products by mass-directed micro-isolation based on prior work (37).

Excitingly, with phthalimide the number of compounds above our typical threshold for isolation more than doubled, from 70 to 187 (see Figure 3B, right). Significant improvements were observed for a wide variety of bromides, including 6-membered ring systems (aryl bromides, pyridines and pyrimidines), 5-membered heterocycles (pyrazoles, imidazoles and thiazoles) and [6,5]- and [6,6]-heterobicycles (indoles, aza-indoles, benzimidazoles, and quinolines). Furthermore, phthalimide improved the resiliency of the reaction, allowing for the tolerance of polar moieties such as 1,2-diols, phenols, and aminopyridines.

We next examined the scope of 384 chemically diverse, relevant carboxylic acids against the structurally complex informers X1, X2, and X13 on nanoscale (1152 total combinations). The phthalimide additive produced significant improvements in a range of primary carboxylic acids including alpha ether, alpha thioether, benzylic and, most notably, non-activated acids, the latter representing a crucial advancement in overall reaction generality as they were previously only narrowly tolerated (Figure 3B left, also see Figure S19, S20, and S21). Further improvements were likewise seen for a range of cyclic carboxylic acids including both activated and non-activated 
4-, 5-, 6-, and 7-membered rings. Gratifyingly, a boost in yield was also seen for a range of acyclic carboxylic acids and 19 of 20 amino acids were cross-coupled successfully in the presence of phthalimide, including both potential sites in aspartic acid and glutamic acid. Finally, the overall functional group tolerance of the reaction appeared far more robust with the addition of phthalimide, with functionalities such as phenols, aldehydes, aryl

\section{A - Benchmarking Phthalimide Against The Informer Library}

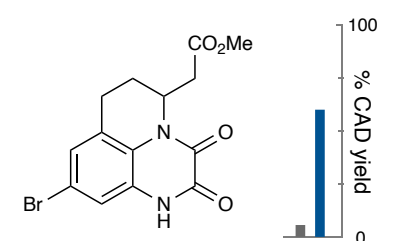

$\mathbf{X} 1$

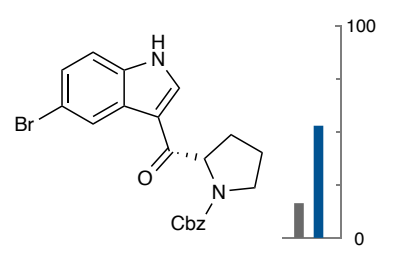

X5

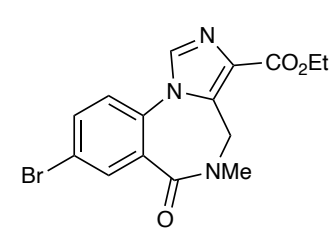

$\mathrm{X} 2$

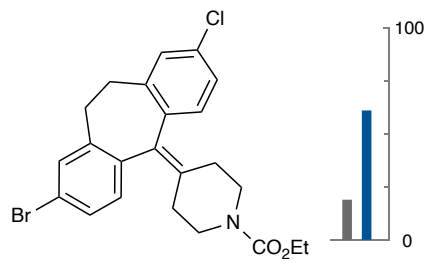

$\mathrm{X} 6$

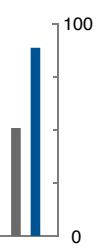

$\longrightarrow \mathrm{N}$

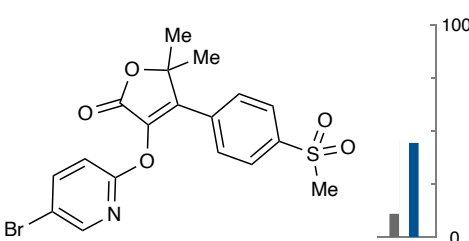

$\mathbf{X 3}$

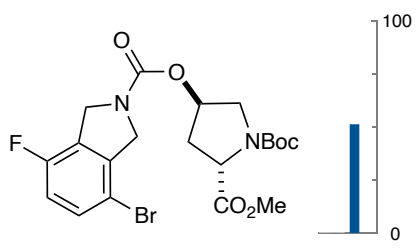

X4

+ Phthalimide

Phthalimide

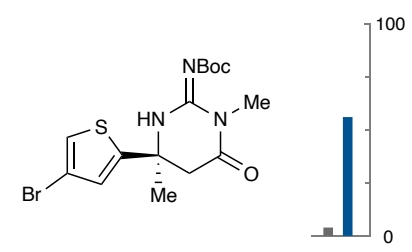

$\mathrm{X} 13$

\section{B - Broad Impact Across Diverse Substrate Classes}

Evaluating 384 Carboxylic Acids Against 3 Informers

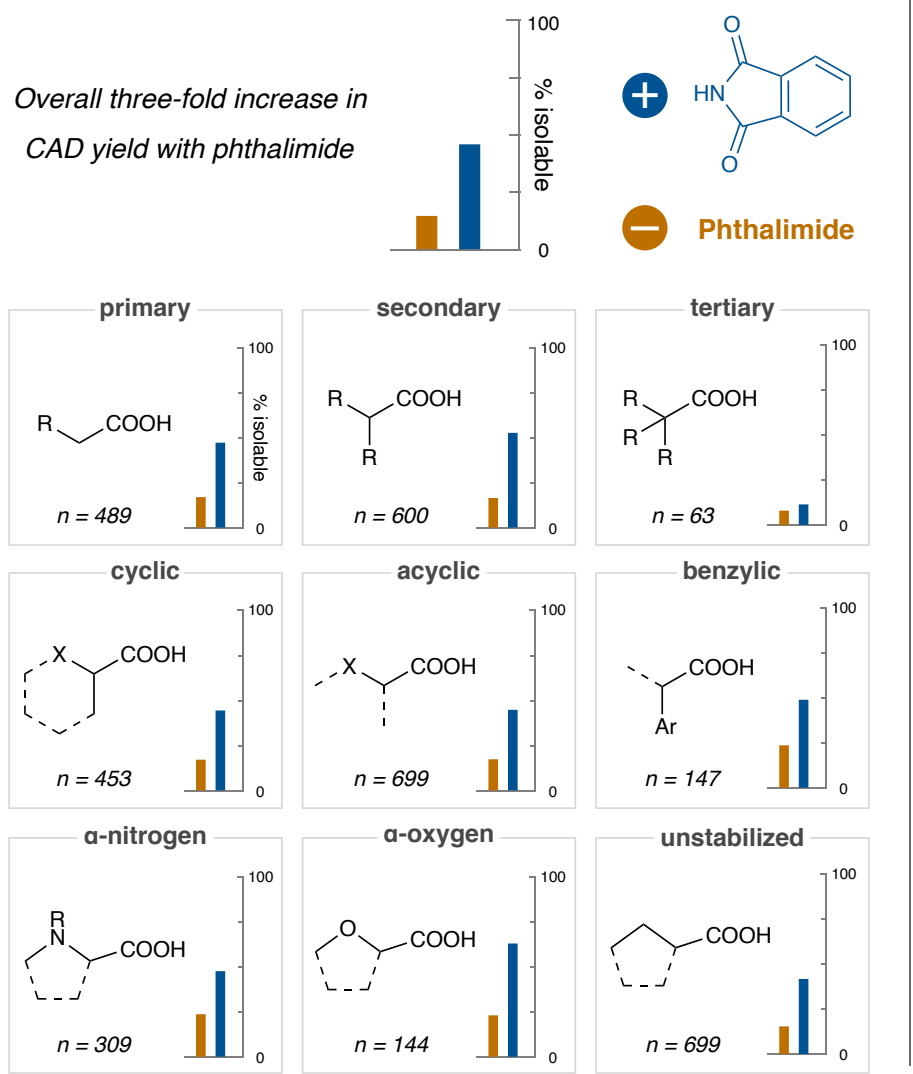

Evaluating 384 Aryl Bromides Against a Complex Carboxylic Acid<smiles>O=C1NC(=O)c2ccccc21</smiles>

Phthalimide

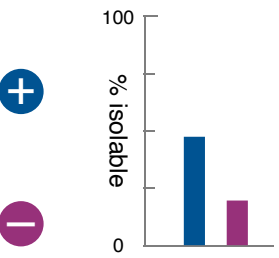

Overall two-fold increase in

$C A D$ yield with phthalimide

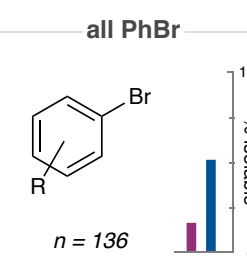

6-mem. heterocycles

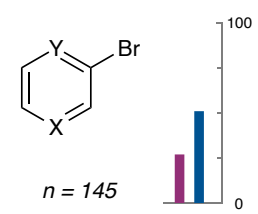

5-mem. heterocycles

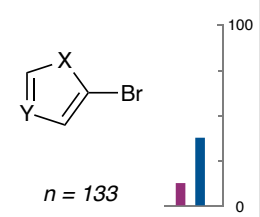

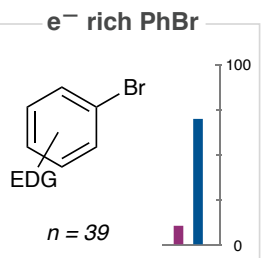

$[6,6]$ heterocycles

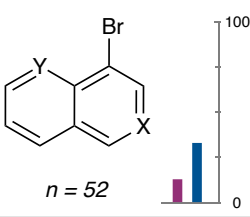

[5,6] heterocycles

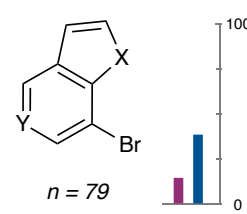

all heterocycles

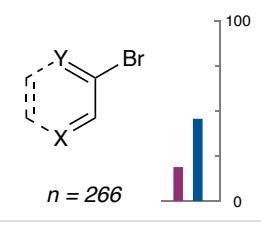

pyridines

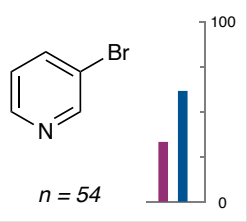

(benz)imidazoles

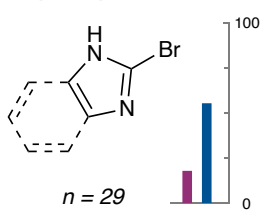

Figure 3 | Scope of the decarboxylative arylation. (A) Scope of the Informer Library investigated against tetrahydropyran-4-carboxylic acid (\%CAD yield reported). (B) Nanoscale high-throughput scope evaluation (\%isolable reported). 
chlorides, and $\beta$-alcohols performing notably better. Overall, in aggregate, the number of reactions delivering products in $>10 \%$ yields increased from 212 to 516 and the overall reaction CAD yield across the set increased more than three-fold. The improvements in both aryl bromide and acid scope observed in this study should, in a realistic setting, have a large impact on the generality of the decarboxylative arylation in synthesis at large.

In line with the principles behind phenotypic screening, previously noted improvements were achieved prior to any detailed mechanistic studies and we next set out to uncover the broader mechanistic implications of the phthalimide additive. It was clear to us from the substrate scope that electron-rich aryl bromides seemed to give particularly strong improvements in yield, and we suspected a correlation between the aryl electronics and phthalimide impact. A Hammett study was conducted both in absence and in presence of phthalimide to probe this hypothesis (Figure 4A, left). A moderately strong Hammett $\rho$ of 1.60 is observed in the absence of phthalimide, which suggests that the oxidative addition of the aryl bromide contributes considerably to the overall rate of the reaction. Satisfyingly, we also found that $\rho$ was nearly fully ablated to 0.58 when phthalimide was added which indicates that phthalimide is favorably impacting the oxidative addition.

We then sought to uncover the specific role of phthalimide in the oxidative addition and evaluated the progress of a model catalytic reaction in presence and absence of phthalimide (Figure 4B, substrate pair yields 10\% w/o phthalimide, $70 \% \mathrm{w} /$ phthalimide). Surprisingly, in the absence of phthalimide the reaction undergoes deactivation over the first 100 minutes to reach an unproductive stationary state, while in presence of phthalimide the reaction steadily goes to completion (see Figure S22 and S23). In conjunction with the Hammett data, we found it reasonable to postulate a deactivation of the reaction due to the formation of low-valent nickel oligomers, which are known to be unreactive towards oxidative addition (38). Independently prepared dimer $[(\mathrm{dtbbpy}) \mathrm{NiBr}]_{2}$ was subjected to the reaction to test this hypothesis (Figure 4A, middle). Trace yield of product was observed with the dimer for the previously used substrate pair, which confirms that this Ni species by itself is incompetent in this reaction. More importantly, repeating this experiment in the presence of phthalimide yields the product in $31 \%$ yield, thus demonstrating that phthalimide can, at least in principle, return unreactive multimers into a catalytically active state. Stable (bpy)Ni $\mathrm{Ni}^{\mathrm{I}}$-phthalimido complexes have been reported which lends support to this hypothesis (39).

Based on these observations, we reasoned that the addition of phthalimide to a deactivated reaction should lead to a reactivation of the catalysts and resumption of product formation. Phthalimide should break up any formed inactive multimeric species and thus allow for productive turnover. In accordance with our hypothesis, when phthalimide is doped into such a reaction we observed almost complete reactivation and steady turnover (Figure 4A, right).

Secondly, we sought to explain how phthalimide turns non-activated acids into competent coupling partners. We observed that non-activated acids tend to lead to large amounts of protodehalogenation in absence of phthalimide and wondered if there were a stabilizing interaction between the putative Ni-aryl complex and phthalimide in the reaction. To emulate the formation of this complex under reaction-relevant conditions (to 
account for carboxylates as likely ligands of the nickel, see Fig 4B for examples), the Ni precatalyst was reduced by dropwise addition of $\left(\mathrm{Cp}^{*}\right)_{2} \mathrm{Co}$ in presence of carboxylic acid, BTMG, and aryl bromide (Figure 4B).

\section{A - Mechanistic Impact 1}
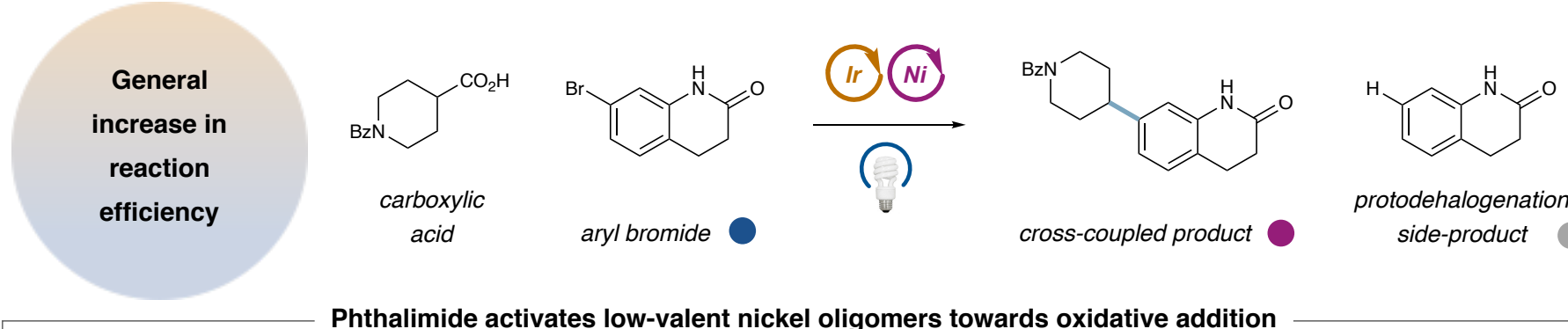

acid

aryl bromide

cross-coupled product side-product

\section{Phthalimide activates low-valent nickel oligomers towards oxidative addition}

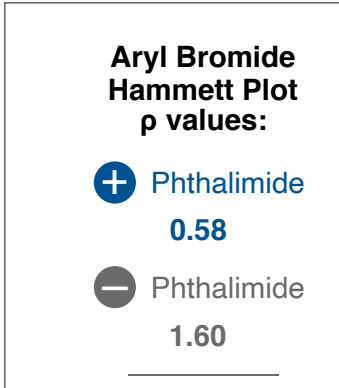

Phthalimide accelerates oxidative addition
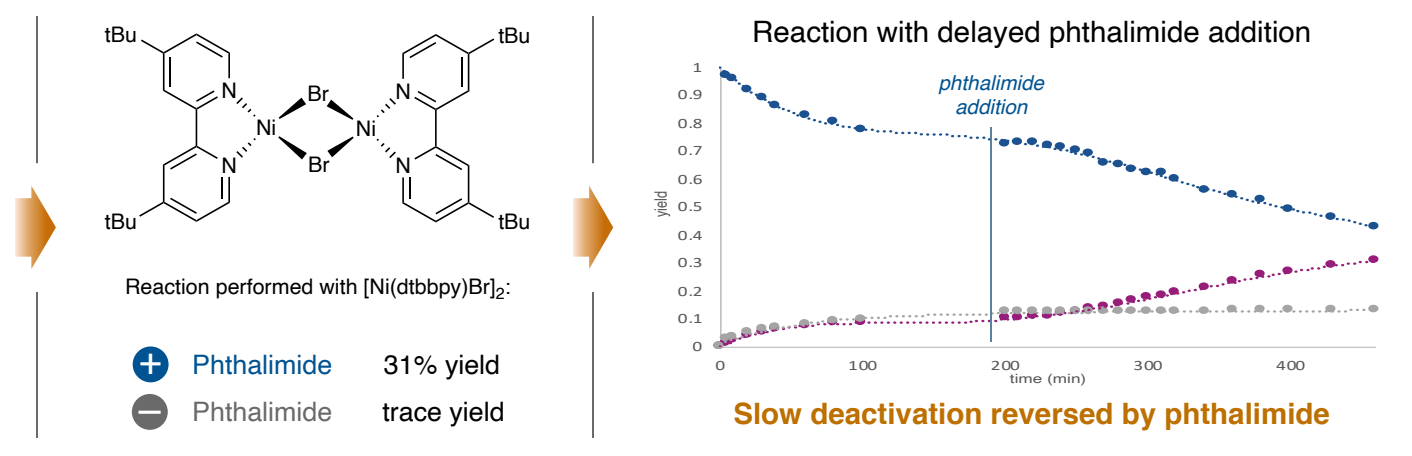

\section{B - Mechanistic Impact 2}
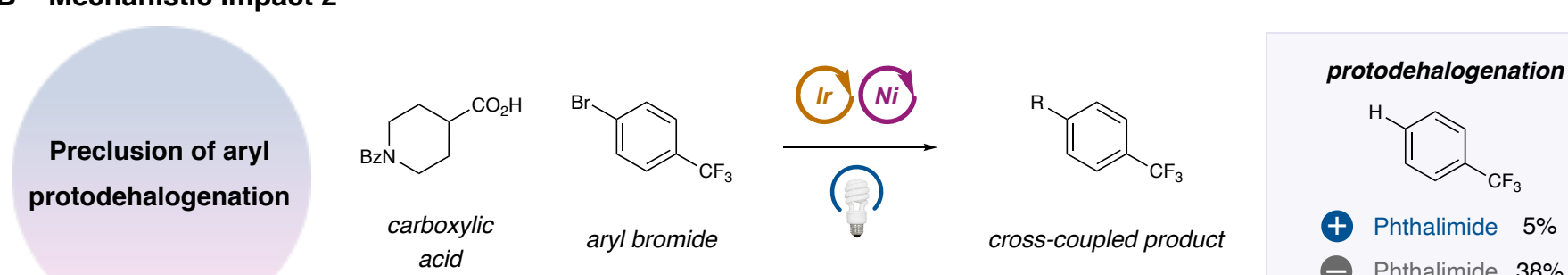

+ Phthalimide $5 \%$
- Phthalimide $38 \%$

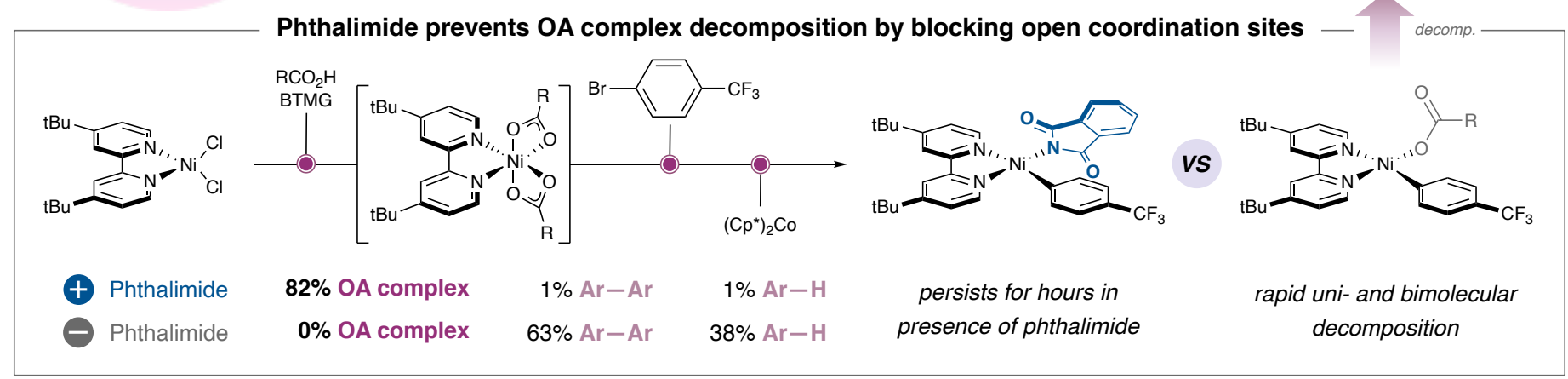

\section{C - Mechanistic Impact 3}

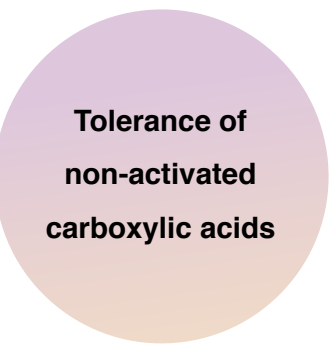

$$
\begin{gathered}
\text { fast for activated acid } \\
(R=N C b z)
\end{gathered}
$$

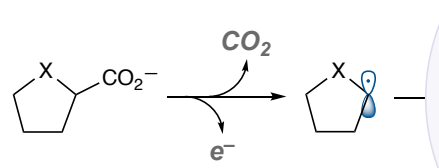

slow for non-activated acid $\left(\mathrm{R}=\mathrm{CH}_{2}\right)$
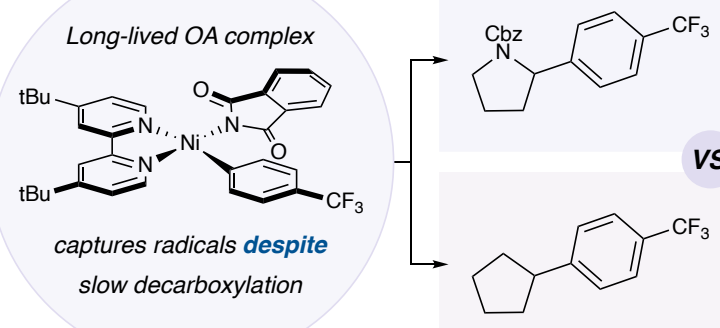

(Phthalimide $91 \%$ - Phthalimide $92 \%$ VS

๑Phthalimide $77 \%$ Phthalimide $23 \%$

Figure 4 | Mechanistic Investigation. (A) Activation of low-valent nickel species. (B) Stabilization of OA complex. (C) Slow decarboxylation now tolerated. 
Excitingly, we found that in presence of potassium phthalimide an oxidative addition complex formed that persisted for several hours, while in absence of phthalimide we only obtained protodehalogenation or biaryl formation. It seems reasonable that the extended lifetimes of oxidative addition complexes allow for the successful trapping of radicals formed from non-activated acids, which tend to undergo comparatively slower decarboxylation (Figure 4C).

Based on the aforementioned experiments, we posit that the Ni cycle is undergoing deactivation due to some off-cycle, unreactive Ni-multimer which results from the aggregation of low valent Ni species. Phthalimide is capable of reactivating said $\mathrm{Ni}$ multimer and allows the catalytic cycle to be productively turned over. Furthermore, phthalimide also appears to impact the stability of Ni-Aryl complexes, presumably by acting as a hemi-labile protecting group that precludes decomposition pathways such as protodehalogenation and aryl metathesis. This also accounts for the inclusion of non-activated acids into the scope of this transformation. We do not rule out further effects of phthalimide in this reaction and additional mechanistic investigations are currently ongoing. The effect of phthalimide on Ni-catalyzed cross-couplings in general is also undergoing further elucidation.

Herein we report a novel method for achieving expedited reaction generality via phenotypic screening. By identifying phthalimide as an ideal additive for the decarboxylative arylation, we were able to develop a general transformation to reliably form $\mathrm{Csp}^{2}-\mathrm{Csp}^{3}$ bonds from feed-stock chemicals. Importantly, this improvement was achieved in less than a year from project inception, which highlights the expedited nature of this approach. Furthermore, in line with the principles behind phenotypic screening the findings reported here yield novel and orthogonal mechanistic understanding of Ni-catalyzed cross-coupling and the authors hope this will give impetus to novel areas of research within this field. We imagine both the use of phthalimide as an additive and phenotypic screening for reaction generality will be rapidly embraced and significantly impact modern organic synthesis.

\section{Acknowledgements:}

The authors are grateful for financial support provided by the National Institute of General Medical Sciences (NIGMS), the NIH (under Award R35GM134897-01), and kind gifts from Pfizer, Merck, Janssen, Bristol-Myers Squibb, Genentech, and Celgene. C.N.P K. acknowledges Princeton University, E. Taylor, and the Taylor family for an Edward C. Taylor Fellowship. J.A.K. thanks the NSF for a predoctoral fellowship (Award DGE 1656466). The content is solely the responsibility of the authors and does not necessarily represent the official views of NIGMS.

\section{Competing Interests:}

D.W.C.M. declares a competing financial interest with respect to the integrated photoreactor. 
References:

1. Brown, H. C. Hydroboration; W. A. Benjamin, Inc: New York, 1962.

2. Tsuji, J. Palladium Reagents and Catalysts; Wiley \& Sons: New York, 1995.

3. Dorel, R.; Grugel, C. P.; Haydl, A. M. Angew. Chem. Int. Ed. 2019, 58, 17118.

4. Trnka, T. M.; Grubbs, R. H. Acc. Chem. Res. 2001, 34, 18.

5. Johansson Seechurn, C. C. C.; Kitching, M. O.; Colacot, T. J.; Snieckus, V. Angew. Chem. Int. Ed. 2012, 51,5062 .

6. Park, Y.; Kim, Y.; Chang, S. Chem. Rev. 2017, 117, 9247.

7. J. A. Lee, M. T. Uhlik, C. M. Moxham, D. Tomandl, D. J. Sall, J. Med. Chem. 2012, 55, 4527.

8. J. G. Moffat, F. Vincent, J. A. Lee, J. Eder, M. Prunotto, Nat. Rev. Drug Discov. 2017, 16, 531.

9. B. K. Wagner, S. L. Schreiber, Cell Chem. Biol. 2016, 23, 3.

10. Brown, D. G.; Wobst, H. J. J. Med. Chem. 2020, 63, 1823.

11. Z Zheng, W.; Thorne, N.; McKew, J. C. Drug Discov. Today 2013, 18, 1067.

12. Swinney, D. C.; Anthony, J. Nat. Rev. Drug Disc. 2011, 10, 507.

13. Pasquer, Q. T. L.; Tsakoumagkos, I. A.; Hoogendoorn, S. Molecules 2020, 25, 5702.

14. Dejonghe, W.; Russinova, E. Plant Physiology 2017, 174, 5.

15. Szabo, M. et al. DDDT 2017, 11, 1957.

16. Ruiz-Castillo, P.; Buchwald, S. L. Chem. Rev. 2016, 116, 12564.

17. Ingoglia, B. T.; Wagen, C. C.; Buchwald, S. L. Tetrahedron 2019, 75, 4199.

18. A. L. Casado, P. Espinet, A. M. Gallego. J. Am. Chem. Soc. 2000, 122, 11771.

19. Vantourout, J. C. et al. J. Am. Chem. Soc. 2017, 139, 4769.

20. Dong, Z.; Wang, J.; Dong, G. J. Am. Chem. Soc. 2015, 137, 18.

21. Bellomo, A.; et al. Angew. Chem. Int. Ed. 2012, 51, 6912.

22. Lovering, F.; Bikker, J.; Humblet, C. J. Med. Chem. 2009, $52,6752$.

23. Walters, W. P.; Green, J.; Weiss, J. R.; Murcko, M. A. J. Med. Chem. 2011, 54, 6405.

24. Jana, R.; Pathak, T. P.; Sigman, M. S. Chem. Rev. 2011, 111, 1417.

25. Han, C.; Buchwald, S. L. J. Am. Chem. Soc. 2009, 131, 7532.

26. Fleury-Brégeot, N.; et al. J. Org. Chem. 2012, 77, 10399.

27. Li, L.; Zhao, S.; Joshi-Pangu, A.; Diane, M.; Biscoe, M. R. J. Am. Chem. Soc. 2014, 136, 14027.

28. Kirchhoff, J. H.; Netherton, M. R.; Hills, I. D.; Fu, G. C. J. Am. Chem. Soc. 2002, 124, 13662

29. Hills, I. D.; Netherton, M. R.; Fu, G. C. Angew. Chem. Int. Ed. 2003, 42, 5749.

30. Brown, D. G.; Boström, J. J. Med. Chem. 2016, 59, 4443.

31. Roughly, S. D.; Jordan, A. M. J. Med. Chem. 2011, 54, 3451.

32. Zuo, Z. et al. Science 2014, 345, 437. 
33. Dombrowski, A. W. et al. ACS Med. Chem. Lett. 2020, 11, 597.

34. Evans, R. W. Novel Coupling Reactions Utilizing Base Metal and Photoredox Catalysis, PhD

Dissertation, Princeton University, Princeton, NJ, 2017.

35. S. E. Dreher, S. W. Krska. Acc. Chem. Res. 2021, 54, 1586.

36. Products were identified via mass spec and UV210 and UV250 signatures. Selected examples were scaled up to confirm product formation (see SI).

37. K.D. Dykstra, et al. ACS Med. Chem. Lett. 2021, 12, 337-342.

38. M. M. Beromi, G. W. Brudvig, N. Hazari, H. M. C. Lant, B. Q. Mercado. Angew. Chem. Int. Ed. 2019 , $58,6094$.

39. B. Nestler, E. Uhlig. Z. anorg. allg. Chem. 1985, 530, 196. 